\title{
It is Not All Fun and Games: Breaking News Consumption on Snapchat
}

\author{
Taryn Bipat*, Tom Wilson*, Ostin Kurniawan, Yoon Jae (Stephanie) Choi \& Kate Starbird \\ Human Centered Design and Engineering \\ University of Washington, Seattle \\ \{tbipat, tomwi, ostin, yoonjc24, kstarbi@uw.edu\} \\ * These authors contributed equally to this work
}

\begin{abstract}
Snapchat is a camera and ephemeral messaging application popular among young adults. Due to its self-destructing content and playful features, Snapchat is often associated with more trivial uses. However, the platform has added functionality to support consumption of news. To understand how users perceive and interact with news content on Snapchat, we conducted semi-structured interviews with 19 users of the platform, focusing on their use of Snapchat during breaking news events, including the 2016/2017 US presidential election and inauguration. Through the lens of Network Gatekeeping, our research explains how users consume breaking news content on Snapchat. We unpack users' ambiguous perceptions of news reliability on Snapchat, and demonstrate how this contrasts with traditional news consumption. Our research also describes how users' mental models of how Snapchat works-specifically their theories about how the platform curates news content-shape their judgments of reliability, media bias and authenticity.
\end{abstract}

\section{Introduction}

Snapchat is a popular camera and messaging application. As of December 2016, it had 153 million daily active users [9]. With playful affordances including face filters and ephemerality, Snapchat has become a lightweight platform that is particularly prevalent among young adults. Among Snapchat users, those aged 25 and under spend on average 30 minutes per day on the platform [9].

Distinctive features of Snapchat include filters and stickers, and the temporary nature of content is often associated with playful uses [36], e.g. sharing selfies [27], mundane experiences [2], and sports fandom [4].

Snapchat introduced other affordances including: Personal (My) Stories (in 2013)—semi-public personal collections of snaps that users have posted; Live Stories (in 2014) — collections of snaps relating to an event that are curated by Snapchat; and Discover
Stories (in 2015)—stories compiled by mainstream media and creative organizations. With the addition of these affordances, Snapchat became a place where news can be created, curated, and consumed.

Since $54 \%$ of young adults aged 18-29 get their news digitally via websites or applications [23], it is important to understand how users consume and perceive news on Snapchat. In this work, we explore the consumption of breaking news through the lens of Network Gatekeeping Theory (NGT) [1,35]. Gatekeeping refers to the control in information systems, traditionally described as an information selection process conducted by a gatekeeper on the gated (i.e. the audience). NGT describes a bidirectional relationship between the gatekeeper and the gated in which both parties can create information and shape its flow [35]. As a conceptual model, NGT provides a lens through which to view social media in terms of information dissemination.

Our work was conducted in the shadow of the 2016 US Presidential Election, a time of political tension and accusations of "fake news" that were directed at various media, including "mainstream" media [31,37]. Our research questions concern how the features and affordances of Snapchat facilitate news consumption, and whether the platform's users consider it reliable. Snapchat's popularity, coupled with new trends regarding news consumption that are perhaps leading to a population less well-informed (and less trusting), provide motivation for studying how Snapchat (and similar platforms) fit into this media environment.

To explore these questions, we conducted semistructured interviews with individuals that used Snapchat during breaking news events. We find that Snapchat is used for the consumption of news, and reveal interesting perceptions of the reliability of news found on the platform.

\section{Background}

Since its release in 2011, Snapchat has provided users with private messaging capabilities with their 
Snapchat friends. Friendships on Snapchat are reciprocal and when adding someone as a friend the other person must accept, leading to a network of closer ties. Photo or video messages - snaps - can be privately sent to selected Snapchat friends. Once opened by the receiver, the snap is visible for just a few seconds, predetermined by the sender, before it disappears. Since 2013, receivers can view the content a second time before it is deleted. More recently, Snapchat began to introduce Stories, described below.

\subsection{Snapchat Stories}

My Stories-User-Produced and Published: Collections of snaps that a user has posted in the past 24 hours - a personal narrative of a person's day. A user's My Story is semi-public, shared only with friends. They are also persistent-Snaps added to My Story can be viewed multiple times and remain visible for 24 hours, after which they are deleted. Users have control over their content in My Story.

Live Stories-Crowd-Sourced PlatformCurated Content: Snapchat Live Stories (also called Our Story) are collections of snaps that are created and uploaded by users and curated by Snapchat in what has been termed a "real-time crowd sourced documentary" [19]. Curation of the Live Stories is done by a team of employees at Snapchat who evaluate user-submitted content and select it for inclusion in the story. As part of this process, the team verifies facts and add additional information to provide context [3]. Around 50-60 snaps make it into each Live Story, but curators select from up to 20,000 submitted snaps [19]. The Live Stories are not ephemeral in the same way as the My Story and direct snaps are-the Snapchat privacy policy makes it clear that content submitted to any of their inherently public features such as Live Stories may be retained as long as necessary [33]. Unlike other social media platforms, Live Stories are hyperlocal-Snapchat users must be within the geographical region of an event to contribute content to its Live Story.

Discover Stories-Media-Curated and Published Content: Collections of snaps compiled by editorial teams, including those representing mainstream media organizations such as the Washington Post, CNN, and ESPN. Discover Stories are available for 24 hours and can be viewed multiple times before they are removed-"because what's news today is history tomorrow" [32]. Snap Inc has recently expanded its Discover channel to feature live hand-curated daily news shows in the platform's vertical format (referring to the aspect ratio)-e.g. MSNBC's Stay Tuned [24], and CNN's The Update [8].

\subsection{Social Media Use During Breaking News Events}

Breaking news refers to developing and nonroutine events. On more traditional media (e.g. television), breaking news has been described as event-driven and based upon unpredictable or unexpected events [18]. However, the modern-day, 24-hour news cycle has led to arguments that breaking news has become more about branding - i.e. breaking news is simply routine news reframed as 'breaking'resulting in fewer independent news stories being broadcast and less well-informed audiences [17].

With the rise of the internet and smartphone usage, it was reported in 2016 that $72 \%$ of adults in the US use mobile devices to access news, and $18 \%$ often use social media to access news [23]. Research into breaking news on social media has looked at Wikipedia [15] and Facebook [14,26], but often focuses on Twitter due to the public availability of digital trace data [e.g. 4,11,13].

\subsection{Social Media: Disruption of Traditional Models of News Production and Consumption}

Social media changed how news is produced and consumed during breaking news events $[10,11,30]$. At the dawn of the social media era, Gillmor explained that new technologies for sharing information online were enabling citizens to report for themselves from the scene of events-i.e. performing as citizen journalists [10]. Following this observation, the widespread adoption of social media in the subsequent decade has drastically altered the model of "news"including who produces it, how it is distributed, who gets paid for it, and how and where people consume it. Some have argued that these changes have contributed to a "crisis in journalism" $[11,30]$, where traditional media outlets are struggling to adapt their practices and business models to new conditions. This "democratization" of news production [10] has allowed new and different groups of people to have their voices heard, such as on Snapchat-which is new, offers unique affordances, and is introducing features that allow for news to be created and consumed in interaction with its distributed users.

\subsection{Distinctiveness of Snapchat as a Platform}

Snapchat differs from other social media platforms (e.g. Twitter, Facebook) in several key ways. First, there is no tagging, such as the hashtag system used by Twitter, so snaps are not grouped by theme or by 
author-i.e. there is no hyperlinked tag that connects users with related content. Second, there is no informal recommendation system (i.e. a "like" on Facebook or "favorite" on Twitter). Although users are aware that content has been viewed, users are not afforded the ability to 'like' something and content is not shared to others who are associated with the 'liker' or the content. Third, there is no native way to share content (i.e. a Facebook share or Twitter retweet) — so a user is not able to forward content to a friend with the click of a button. This leads to interesting and understudied questions about news consumption in a markedly different social environment.

\section{Methods}

Our research used a grounded approach, informed by Strauss and Corbyn [34] and Charmaz [7]. We conducted semi-structured interviews with 19 Snapchat users between March and June 2017. Our methods were qualitative and iterative, with two distinct phases of interview: exploratory and targeted (described below). We used a grounded, interpretive approach for the analysis of the notes taken during the interviews. Through thematic analysis and affinity diagramming, we developed a common set of themes that emerged from these data. Subsequently, we returned to the video and audio recordings of the interviews to identify additional content related to those themes and to transcribe relevant sections and obtain participant quotes to support our findings.

Nineteen participants were recruited between January and June 2017 using flyers posted in the student union building of a large US west coast university. The recruitment poster asked "How do you use Snapchat?" and explained that researchers were conducting interviews "to learn more about users' day to day use of Snapchat". Interested participants were directed to a short online screening survey that asked their age and frequency of Snapchat use (daily, weekly, monthly, once, or never). A follow up email asked potential participants if they had ever used Snapchat during a breaking news event. If so, the interview was scheduled. Our recruitment materials did not refer to 'fake news' or recent political events.

We used interviews as a method to obtain rich, qualitative data about Snapchat use during breaking news events. Our interviews were semi-structured around primarily open-ended questions to facilitate an in-depth exploration of participants' experiences using Snapchat. Each interview lasted up to one hour and was carried out by two researchers - one followed the interview protocol while the second took notes and considered additional questions to help probe deeper into participants' experiences. Interviews were video and/or audio recorded and later transcribed.

Interviews were conducted in person $(n=17)$, or using Google Hangouts $(n=2)$. The median age of participants was 19 (only one participant was older than 25). A majority of participants used Snapchat on a daily basis $(n=17)$, one weekly and one monthly. These demographics are consistent with a report by Snap that shows, on average, Snapchat users aged $<25$ spend 30 minutes on the platform every day [9].

In the exploratory round (P1-P14), our interview protocol was designed to elicit participants' use of Snapchat at various times and in a variety of situations. We began by asking about their most recent and most memorable use of the platform, and then focused on their use of Snapchat during an emergent or breaking news event of their choice. We finally asked specifically about their use of Snapchat during recent political events, including the 2016 US Presidential Election, 2017 US Presidential Inauguration, and related protests. During our interviews, we explicitly asked participants about reliability of news on Snapchat to gauge whether users thought it provided a consistent account of events, when compared to other sources.

After analyzing our first 14 interviews we refined our interview protocol, adjusting it to focus specifically on the themes that had emerged as salient to the research in the exploratory round. This approach is consistent with grounded theory, which advises researchers to follow the theoretical direction of their data and reconsider the protocol for subsequent interviews [7]. We conducted five more interviews (P15-P19) with our refined protocol that focused on the issues of reliability of news content on Snapchat.

\section{Findings}

Through our interviews with Snapchat users we discovered that although the interactions on platforms like Snapchat have often been viewed as fleeting and trivial [e.g. 3,17,24], people are also using these platforms for the more "serious" activity of consuming breaking news-albeit with variations in the perceptions of reliability.

Some participants described consuming "news" through their Snapchat friend networks. However, the majority of participants reported that their primary news consumption on Snapchat took place through accessing Live Stories and Discover Stories, suggesting users valued the curation processes that are conducted by Snap Inc (Live Stories) or media organizations (Discover Stories). 


\subsection{Consuming News through Discover Stories}

Through its Discover Stories Snapchat serves as an intermediary that gives access to more traditional news content-and effectively selects which sites and stories will be accessible to users through their platform. In our interviews, participants noted using Discover Stories to consume news from the featured news organizations. Some explained how they used Discover Stories as a replacement for televised or online news. P12 used Discover Stories as a way to keep her informed of current events, for example during a recent natural disaster she obtained information from Snapchat:

"I don't watch the [televised] news much so when I'm scrolling down on Snapchat [Discover Stories] usually inform me about how many people were injured, what was happening, where it was." (P12)

Snapchat provided a gateway to news content that P12 may not otherwise encounter. However, P12 was an outlier-most of our interviewees positioned Discover Stories as a supplement, rather than a replacement, for more traditional sources of news.

\subsection{Consuming News through Live Stories}

Live Stories are collections of crowd-sourced content-uploaded by users to be included in publically visible stories. However, before going public, this content is curated by Snap Inc. Since Snap Inc makes the final editorial decision as to which usergenerated snaps appear on the Live Story, they are performing a hybrid form of gatekeeping-i.e. controlling the information that flows through their platform [1], and selecting the voices that are heard and the perspectives that are shared.

\subsubsection{Opening a Window to Events on the Ground}

In our interviews, Snapchat was rarely the initial means of discovering an event-participants consistently described encountering the information elsewhere first - but Snapchat was a place that users went to see content in a specific format and style. In particular, Snapchat's support for short video content provided a certain type of display, a window into events on the ground, that users appreciated.

"I read the news, and if Snapchat presents content that is related to the news that I read, I see it. It kind of gives me a visual component to what I've read. A witness perspective. Because the person who was Snapchatting is actually there. So that's interesting." (P15)

The crowd sourced content of Live Stories is used to document current events, helping to inform
Snapchat users about emergent events at a specific location, providing viewers with a 'boots on the ground' perspective, through the eyes (or smartphones) of affected individuals. Live Stories are perceived as offering something more than the content captured by mainstream media:

"I would have known about it [event] but I wouldn't have known what was happening so close to it if people were not Snapchatting about it...I was watching it through Snapchat, I wasn't really watching it anywhere else but I was reading articles on Twitter." (P17)

Although participants often had access to news through other media (e.g. TV, online news) or platforms (e.g. Twitter, Facebook), they found a specific value in consuming news through Snapchat because they were able to get a better understanding of exactly what was happening in close proximity to the event from a first-person perspective.

\subsubsection{Reintroducing Forgotten News}

Participants also noted that Live Stories provided 'airtime' for issues that had otherwise dropped off the regular news cycle of the mainstream media. For example, P14 recalled a specific Live Story that was available during a debate in Michigan during the 2016 US Presidential Election:

"One of the debates was in Flint, Michigan, I used the Live Stories function. There were debaters and commentators giving their opinions...That the

Flint water crisis was still going on but we completely forgot about it is horrifying." (P14)

Although Snap Inc. ultimately curated the story; it is likely that a critical mass of user-generated content pushed Snap to publish the Live Story. In this sense, Snapchat users have some power to set the agenda on Snapchat-i.e. to identify issues that are important to them, which is exercised by submitting snaps to a Live Story. However, since Snap Inc controls the flow of information onto the final story (i.e. through selection, shaping, and localization), Snapchat users remain bounded in a relationship that is not balanced [1].

\subsubsection{Hyper-Localizing News with Geo-filters}

Snapchat monitors users' locations and depending on where they are the application will offer localized filters, in the form of banners that appear at the bottom of the snap, providing users with additional contextual information - the geographic origin of the content. This explicit geotagging is something that users particularly enjoyed about the coverage of the 2017 Presidential Inauguration protest marches: they could view various perspectives of breaking news events. 
The geotagging feature also applies to uploading a snap to a Live Story - users must be proximal to an event to contribute content, ensuring that snaps originate from people 'on the ground', offering a hyper-local perspective that interviewees appreciated.

\subsection{Perceptions of News Reliability on Snapchat}

\subsubsection{Conflicting Perceptions of Reliability}

In each interview, we specifically asked participants if they thought the content they viewed on Snapchat was a reliable form of news. We received mixed responses depending on whether they discussed Live Stories or Discover Stories, but also discovered interesting contrasts within each.

Across the participants, there was disagreement about whether or not the user-generated and platformcurated content on Snapchat was a reliable source for news. Some participants believed that because Snapchat is not the only source of news, the reliability does not matter-ultimately, the user must decide whether they think it is reliable or not:

"Everyone can upload videos about news. Even though it is not $100 \%$ accurate at least you can get what the idea is. As a viewer, you have the responsibility to make an educated guess of what the content is. [It is not $100 \%$ accurate] because it is based on human nature to record something, not research." (P19)

P19's judgment of reliability was based on their preconceived ideas of news and what a typical Snapchat user should perceive news to be. However, other participants formed their reliability judgments by comparing Snapchat content to news from sources such as television and other social media. P19 suggested that the selection of those selected to be on Live Stories is more real than the portrayals in popular media. This suggests that some users view Snapchat as a diverse and balanced news source, more than they may the traditional news organizations.

"You can get a real view of the story because the media usually picks people to interview, but from Snapchat you can view people." (P19)

This comment also speaks to the perception that Snapchat disrupts traditional gatekeeping dynamics, where "mainstream" media get to determine which voices are heard. This is often viewed, as it is here, as a positive development. These comments also suggest, somewhat paradoxically, that Snapchat has stepped up to play a similar role - a networked gatekeeper that gets to select which voices are heard-but is able to do so without eliciting the same kind of criticism assigned to traditional news media.

Since Live Stories are Snapchat-curated collections of user-generated snaps, Snapchat is able to select and unite information to form its chosen narrativetherefore assuming an information control activity of a gatekeeper [1]. Although P9 did not see Snapchat as a reliable source of news, she concurred with P4 that it was a good channel to obtain various perspectives:

"[It's] not a reliable form of news, but could be good for getting different people's perspectives of a story." (P9)

P4 thought that the curation of Live Stories helped make it more interesting and "kind of" unbiased. Referring to a perceived bias present in the mainstream media, P4 viewed the coverage offered by Snapchat Live Stories as offering a more balanced view of the news - in this case, from both ends of the political spectrum:

"It is really interesting to see different people's perspectives, very quick raw opinions that people have...it is curated but I think they do a nice job of making it interesting and kind of unbiased. On election night you saw people on completely different ends of the spectrum celebrating or really defeated" (P4)

\subsubsection{Reliability Implications, Loss of Journalistic Voice}

We found users struggling with the dilemma of removing the journalistic voice from news production. For example, P19 held conflicting views about the reliability of live sources. While she believed it was good to have the opinion of the general public, she also felt that Snapchat could not be the only source of news because the content does not come from experts.

"I don't think Snapchat is enough [as a sole source]. Even though it's from real people, they're not experts on the news...I need sources from experts, too. " (P19)

Similarly, P15 believed that only people trained as journalists could contribute reliable news:

"No [I don't see Snapchat as a reliable source of news]. I don't trust the public's intelligence [laughs]...with actual journalism, then it's people who know how to write, fact check things-I expect them to fact check things, whereas people on Snapchat are presenting what they see, which is interesting, but I don't think their opinion is valid. I like to see what they are seeing but I don't take into account their personal opinions." (P15)

In these examples, users are assessing the reliability of the content they find on Snapchat by drawing comparisons with the norms of traditional news, where trained experts create stories. Since people not trained in journalism could produce Live 
Story content, some users remain skeptical about its reliability, despite the curation process at Snapchat.

\subsubsection{Reliability a Function of the Content Curation Process}

For many participants, their perceptions of reliability as a news source were connected to their understanding of how the content was curated. P2 distinguished between the credibility of individual snaps [of the Live Story] and the actual Live Story. They believed that the snaps themselves were credible as they were not filtered or edited. However, Snap's Live Story selection process was opaque and thus the reliability uncertain:

"Live Stories are helpful because you see something happening live and it's not really filtered or edited. But you can think about if people choose what's posted on the Live Story. That could be accurate or how true to telling this story." (P2)

P2 speaks to the perception of Snapchat being unfiltered news. Taken in the context of rhetoric about media bias and "fake news" [31,37], this comment stands out as identification of Snapchat as a potentially more "real" channel for news, although that could be undermined by bias in the curation process.

Similarly related to the concurrent discourse about media bias, P5, believed that media organizations on Discover Stories were on the platform for profit rather than to share valid information:

"Probably not, because it is from the mouths of people that are either looking to sell people things, you know like Buzzfeed or the magazines, or like from real people who have biases." (P5)

These participants based their perception of reliability on the reputation of the content provider, not Snapchat, which took the role of a hosting platform. Directly speaking of "fake news", and in contrast to P2, P12 believed that the reliability of the user generated content was due to it being reviewed by Snap Inc. She perceived the curation process as improving reliability, commenting that she had not seen any "fake news" on Snapchat Live Stories:

"I think [they are reliable] because everything needs to be reviewed before. Besides photos and videos they also include captions and descriptions. And I haven't encountered any that was like, fake news." (P12)

For P12, the curation process of Live Stories means that the content met a certain threshold to be accepted and is enhanced with additional material such as captions, adding value to the content. P12 also explained that the first person format improved her experience of Live Stories, suggesting that authenticity contributes to a sense of reliability or credibility: “...it's kind of nice to see them talk directly to the camera using their own phone rather than speaking to a camera that someone is holding for them." (P12)

Focusing on the same dynamic of raw data sharing, P11 asserted an opposite view-Snapchat was not a reliable source because taking chunks of content out of context was a problem that disrupted the credibility:

"A lot of the things that people post are very small sound bites, and when you take something out of context ...People tend to do that. And when you take things out of context it disturbs the reliability." (P11)

A point to note here is that traditional media such as the radio and television also make use of sound bites that can be taken out of context, but P11 does not mention this in his criticism of Snapchat. This may speak to the perceived credibility of the Snapchat, rather than to the specific content shared there.

\subsubsection{Reliability Tied to User Perceptions of Source}

Some Snapchat users turn to Discover Stories to access content provided by "legitimate" news producers. Perceptions of reliability on this channel were often tied to the perceived reliability of the upstream source:

"I would say it's more reliable than what I see on Facebook because I do not think my friends would post fake news, and the stuff on Discover Stories is from legitimate news or media agencies...I don't think they would risk posting junk on their Snapchat handle." (P7)

In this example, P7 draws comparisons with Facebook, a platform that was publically called out (in 2016) for facilitating the distribution of "fake news" $[31,37]$. Referring to his friends' My Stories, P7 explained that he was confident that they would not post "fake news", which he felt was a problem on Facebook. Continuing on, he references Discover Stories, and explains that he viewed the sources of those stories as legitimate and unlikely to post content of questionable reliability.

Similarly, P10 spoke of the reliability of the sources of the Discover Stories. He viewed Snapchat (generally) as a reliable platform for viewing the content, but when considering the reliability for a specific story, that was dependent of the source:

"I would say that it's reliable if it's from the [Discover Stories], some reliable websites...it's reliable but it depends on who it's coming from." (P10)

P15 explained this dependency on this source by noting that their perceived reliability of content was due to the curation process being conducted by editors from traditional news organizations: 
"I think [Discover Stories] is more reliable [than Live Stories] because I know there are editors behind those stories. Cosmopolitan usually has one op-ed, especially after political events, and I know who the person writing is as I can just google her. So I like reading those op-eds. Sometimes Buzzfeed has a couple like "here's what I learned in life" and because I know who that author is I can trust that they're not bullshitting." (P15)

A few participants shared this view-they had more trust in the Discover Stories because the curation process was similar to traditional models, whereas the Live Story curation process remained opaque.

\subsubsection{Reliability Impact of Snapchat's Playful Reputation}

Other participants determined the reliability of news on Discover Stories based upon their perceptions of Snapchat. These participants believed that content on Discover Stories was essentially an extension of what Snapchat was known for-i.e. more playful and trivial content. For example, P10 discusses that Discover Stories are not meant for content of serious nature, but rather should have entertainment value.

"I always think that Snapchat is for entertainment purposes, for comedy, for jokes...Sometimes the [Discover Stories] will have a serious title but when I click into it, it's a parody, not very serious." (P10)

Similarly, P14 referred to this underlying nature of Snapchat in her assessment of the reliability of the medium. She elucidated that placing news within a small temporal space did not allow the full story.

"...With Snapchat as a medium it [BuzzFeed] doesn't seem as weighted because of the nature of the app...Buzzfeed and others try to reflect that through Snapchat stories, they make them very light...there's a lot of emojis everywhere, they try adapting to the medium but at the same time a message is kind of lost...some stuff is lost in translation and I don't think it's really a viable way for me to get news."

This comment underscores this association of Snapchat with light and playful content, and how that colors some users' perceptions of news credibility, both crowd-generated and media-produced. P14 explained how the stories were sensationalized:

"[Live Stories are] light and not very serious...there are emojis everywhere...they would have titles like ' 10 things you NEED to know about Donald J. Trump or Hillary Clinton'. I didn't really want to click those"

To P14, Discover Stories comprise of clickbait titles and emojis - detracting from the reliability of the news to the extent she would not want to read them.
Similar to their perceptions of Live Stories, Snapchat users judged the reliability of Discover Stories differently. The media curated content in Discover Stories led to perceptions of media bias dependent on the recognition of the news organization. It was compared to more traditional mass media, but based on affordances of the Snapchat medium, the content was more sensationalized than "real news".

\section{Discussion}

News consumption is increasingly facilitated by social media platforms such as Twitter, Facebook, and Snapchat. Due to the "democratization of news production" [13], and the ability for information to propagate (quickly) through and across these sites, often in derivative forms, it has become both increasingly hard and increasingly necessary for consumers to make reliability judgments about "news" content they encounter. Like Facebook and Twitter, the mobile application Snapchat has become an alternative way to access traditional news (through Discover Stories) and a novel way to consume point-of-view crowd-sourced content submitted to Live Stories by other users.

The distinctive affordances of the services provided by Snapchat provide a variety of user perspectives on the consumption and reliability of news. Through NGT, we better understand user perspectives and how they diverge from traditional news consumption. We also show how users' understanding of the Snapchat curation process shape assessments of reliability, media bias, and authenticity.

\subsection{Juxtaposition of Social Media and Traditional News}

Traditional news organizations are represented in Discover Stories, allowing Snapchat users to see current news from e.g. CNN, Buzzfeed, New York Times. Although they consumed news through Live Stories, participants noted their main gateway to news was through Discover Stories, suggesting an attachment to traditional sources of news, even if the technology used to access them is evolving. Some participants believe they are provided with a balanced perspective, and that Snapchat gives an unbiased view through links to external sources and the creation of stories from experts. This allows Snapchat to supply a range of content in a simplified, non-intrusive fashion, with responsibility on the user to find out more.

Snapchat's geotagging feature is another way Snapchat opens the door to more balanced 
perspectives about a particular event. Participants mentioned being able to see events and others' reactions from all over the world. Contrary to the perception that social media produces a polarizing "filter bubble" [25,31,37] whereby users reinforce their own biases, those consuming content on Snapchat believed that they were able to see a more balanced perspective through a distributed view of emergent events. The variety of views from global perspectives and external links reflects 'external diversity' [21], which suggests that the influence of external sources signify that Snapchat need not focus on reporting objectively and impartially, but rather spend more time evaluating that its content is reliable [21]. While this exemplifies Snapchat as a more reliable source of news, there remains ambiguity in what our participants believed this was.

In current public discourse, the term "fake news" has been directed at both the "mainstream" media and the alternative media sites now competing with them. One aspect of this problem is the introduction of clickbait news - news-like content with sensationalist headlines, designed to encourage clicks [5]. This has led, in some cases; to adaptations by more traditional news providers to compete with similarly designed content, making it more difficult to judge reliability of online news generally. Our findings suggest clickbait news may erode the perception of reliability of a platform over time. Underscoring this point and highlighting a challenge for Snapchat as they consider how to incorporate news content into their user experience, some participants appeared to allow their perceptions of Snapchat-i.e. as a playful platform for lightweight communication between friends - to color how they judged the information they found there. Additionally, claims of perceived biases in mainstream media have been made by peopleincluding prominent political figures, who may be employing this as an intentional strategy to diminish the credibility of news sources [28]. Our interviews show Snapchat users, like the rest of us, struggling to understand media biases and to weigh the reliability and credibility of the news content they encounter.

Our findings suggest that, in a somewhat circular way, this ambivalence with the traditional news media has led some people to turn to social media platforms, including the ephemeral content platform Snapchat, for news consumption-rationalizing that these platforms provide more factual or more "real" content. Some of our interview participants view news found on social media as more authentic because it originates from the location of the story, like a journalist's account would, but independent of a large media organization - each of which has its own political leanings, culture, agenda, and business model.
Participants also felt that content found on Snapchat was impactful, and perhaps being outside the influence of a large media organization (as Live Stories are) meant issues no longer featured on regular news could be brought back to the public's' attention.

The contrasting effect occurring between traditional and social media news is apparent in our findings. News-consumption behaviors have developed through past experiences with traditional forms of social media but the different affordances of Snapchat, along with the traditional nature of news consumption, influence the perceptions of reliability of news on Snapchat.

\subsection{Inconsistent Perceived Reliability}

In our interviews, we specifically asked about "reliability", but participant responses reflected perceptions based upon credibility, bias, and authenticity. We unpack these distinct concepts in the following sections.

\subsubsection{Reliability as Credibility}

Discover Stories offered Snapchat users a way to access traditional media sources direct from the platform. Users who felt content within Discover Stories was reliable thought so because large wellknown news organizations contributed informationadding credibility to the news. This is consistent with research on evaluating credibility of online sources through cognitive heuristics [22]. The reputation heuristic states that reputation and name recognition of a source (i.e. a news organization) influence the user's perceptions of credibility, and people choose more recognizable content over less familiar [12].

\subsubsection{Reliability as Bias-Free}

Both Discover and Live Stories are subject to some sort of gatekeeping and consequently influenced by culture, political leaning, and agenda [20]. Two participants reflected positively on Live Stories, saying that they offered a range of perspectives which felt unbiased. At a time when media bias and blatant misinformation were perceived as a major problem with online information - in the wake of the 2016 U.S. general election-Snapchat perhaps provided a refreshing alternative to "mainstream" news, which was viewed at that time as being heavily partisan.

\subsubsection{Reliability as Authenticity}

Another aspect of participant's reliability judgment was their view regarding the authenticity of 
the content. There was an interesting contrast between users who thought Live Stories were more reliable because the content was "not really filtered" (P2), and those who felt they were more reliable since "everything needs to be reviewed before" (P12). This is informative of the current media climate, where talk of "fake news" is eroding the perceived credibility of news organizations and social media platforms.

\subsection{Snap Inc. as a Networked Gatekeeper}

Relevant literature on gatekeeping [1,29,35] provides a lens through which to better understand both news consumption on Snapchat and users' perceptions of reliability. The three story types ( $M y$ Stories, Live Stories, and Discover Stories) demonstrate three different types of gatekeeping.

For personal My Stories, users are their own gatekeepers, creating the content and deciding for themselves who can view it. The platform does not provide additional gatekeeping-for example by filtering, privileging or otherwise controlling information flows.

In contrast, Discover Stories bear a similarity to traditional mass news media and adopt a gatekeeping process that corresponds with early definitions of gatekeeping [29], "the process by which the millions of messages that are available in the world get cut down and transformed into the hundreds of messages that reach a given person on a given day." (p.1). Discover content is created and selected by editors and creatives at media companies, who engage in gatekeeping as they decide what content is produced and published. Snap Inc. explains that the content uploaded to Discover is at the discretion of the corresponding media companies [37]. At a higher level, Snap Inc. performs a networked gatekeeping role as it decides which producers are featured on Discover. NGT [1] describes this as strengthening the relationship between both human and technological networks and the gatekeepers and gated-and the impacts that this has on the gated. We found that conflicting reliability perceptions were based upon feelings about both the media organizations responsible for the content, and Snapchat's role as the overall networked gatekeeper.

Live Stories initiate a bidirectional relationship in which users generate content and Snap performs network gatekeeping actions (e.g. selection, addition, joining, timing, deletion) through its curation. However, this is not a fully reciprocal relationship, and the lack of transparency surrounding the curation process influences users' mental models of the process and shapes their perceptions of reliability. For example, one of our participants reported that content they submitted to a Live Story was edited, with part of it removed to fit into the story. In this case it was "just" footage of a festival, but such practices impact users' perceptions of information credibility on the platform.

Analyzing our findings through the lens of NGT revealed the nuanced relationships between gatekeepers and gated across the different "story" types. These varied dynamics help explain the ambiguous perceptions of reliability relayed to us by our participants. Users' perceptions of reliability were shaped by their understandings - or mental modelsof how information was generated, curated and delivered to them. This in turn extended into their experience with, or perception of, the different organizations (and individuals) who played various roles within this process. For Discover Stories, the top level had news organizations as gatekeepers. For Live Stories, the gatekeepers are perceived both as the users who contribute the content (with mixed opinions on whether this can be viewed as reliable) and curators who work at Snapchat (with very different conceptions of who these curators are and how they work).

\section{Limitations}

We recruited exclusively from a student population. Although this demographic aligns with Snapchat's predominate user base, most of our participants were from a single university. Second, Snapchat is constantly evolving and features were altered during our research. Thus users' understandings and impressions of the platform were rapidly changing and were in some cases underdeveloped - i.e. not every participant was aware of all the functionality available to them, specifically around the news distribution features.

\section{Conclusion}

People are turning to social media for news consumption leading to widespread attention about the reliability of news content found there. Our study aimed to understand how Snapchat is used to consume news and how users perceive the reliability of news content, and was conducted in the shadow of the 2016/2017 US Presidential Election cycle, during political tensions and accusations of "fake news". We found that Live Stories and Discover Stories afford different ways of users perceiving the reliability of the information. Participant's perceptions of the reliability of Live Stories were connected to their understanding of how content was curated; the perceptions of Discover Stories aligned with users' understanding of the source, with the reliability depending on the 
credibility of the organization posting the content. This research improves our understanding of Snapchat use during breaking news events and how it contributes to perceived media bias and reliability.

\section{References}

[1] Karine Barzilai-nahon. 2008. Toward a Theory of Network Gatekeeping : A Framework for Exploring Information Control. 59, May: 1493-1512.

[2] Joseph B. Bayer, Nicole B. Ellison, Sarita Y. Schoenebeck, and Emily B. Falk. 2015. Sharing the small moments: ephemeral social interaction on Snapchat. Information, Communication \& Society 4462, January: 1-22.

[3] BBC. 2017. Snapchat: We call police to check facts. Retrieved August 1, 2017 from

http://www.bbc.com/news/technology-41085930

[4] A. C. Billings, F. Qiao, L. Conlin, and T. Nie. 2015. Permanently Desiring the Temporary?: Snapchat, Social Media, and the Shifting Motivations of Sports Fans. Communication \& Sport [5] Jonas Nygaard Blom and Kenneth Reinecke Hansen. 2015. Click bait: Forward-reference as lure in online news headlines. Journal of Pragmatics 76 [6] Axel Bruns and Tim Highfield. 2012. Blogs, twitter, and breaking news: The produsage of citizen journalism. In Produsing theory in a digital world: The intersection of audiences and production. 15-32. [7] Kathy Charmaz. 2006. Constructing grounded theory: a practical guide through qualitative analysis. [8] CNN. 2017. CNN Launches New Daily News Show for Snapchat: "The Update." CNN. [9] SECURITIES AND EXCHANGE COMMISSION. 2017. FORM S-1 REGISTRATION STATEMENT UNDER THE SECURITIES ACT OF 1933 for Snap Inc.

[10] Dan Gillmor. 2004. We the Media: Grassroots Journalism by the People, for the People. O'Reilly [11] J. Fuller. 2010. What is happening to news: The information explosion and the crisis in journalism. University of Chicago Press.

[12] G. Gigerenzer, P. M. Todd, and ABC Research Group. 1999. Simple heuristics that make us smart. Oxford University Press.

[13] Dan Gillmor. 2006. We the Media: Grassroots Journalism By the People, For the People.

[14] Alice Ju, Sun Ho Jeong, and Hsiang Iris Chyi. 2014. Will Social Media Save Newspapers?

Journalism Practice 8, 1: 1-17.

[15] Brian Keegan, Darren Gergle, and Noshir Contractor. 2013. Hot Off the Wiki: Structures and Dynamics of Wikipedia's Coverage of Breaking News Events. American Behavioral Scientist 57, 5: 595-622. [16] Haewoon Kwak, Changhyun Lee, Hosung Park, and Sue Moon. 2010. What is Twitter, a Social Network or a News Media? IW3C '10): 1-10. [17] Justin Lewis and Stephen Cushion. 2009. An analysis of breaking news stories and their impact on the quality of 24-hour news coverage in the UK.

[18] STEVEN LIVINGSTON and W. LANCE
BENNETT. 2003. Gatekeeping, Indexing, and LiveEvent News: Is Technology Altering the Construction of News? Political Communication 20, 4: 363-380.

[19] Victor Luckerson. 2015. How Snapchat Built its Most Addictive Feature. Time. Retrieved from http://time.com/4049026/snapchat-live-stories/ [20] Maxwell E McCombs and Donald L Shaw. 1972. The Agenda-Setting Function of Mass Media. The Public Opinion Quarterly 36, 2: 176-187.

[21] Denis McQuail. 1994. Mass Communication Theory.

[22] Miriam J. Metzger and Andrew J. Flanagin. 2013. Credibility and trust of information in online environments: The use of cognitive heuristics. Journal of Pragmatics 59: 210-220.

[23] B Y Amy Mitchell, Jeffrey Gottfried, Michael Barthel, and Elisa Shearer. 2016. Amy Mitchell, Jeffrey Gottfried, Michael Barthel and Elisa Shearer. [24] NBC News. 2017. NBC News Launches 'Stay Tuned,' a Snapchat News Show.

[25] Tien T Nguyen, Pik-Mai Hui, F. Maxwell Harper, Loren Terveen, and Joseph A. Konstan. 2014. Exploring the filter bubble. $W W W^{\prime}$ '14: 677-686. [26] Miles Osborne and Mark Dredze. 2014. Facebook , Twitter and Google Plus for Breaking News: Is There a Winner? ICWSM '14: 611-614. [27] Lukasz Piwek and Adam Joinson. 2016. "What do they snapchat about?" Patterns of use in timelimited instant messaging service. Computers in Human Behavior 54: 358-367.

[28] L. Qiu. 2017. Fact Check: Trump Blasts 'Fake News' and Repeats Inaccurate Claims at CPAC. The New York Times.

[29] Pamela J Shoemaker. 1991. Gatekeeping. [30] Ignacio Siles and Pablo J. Boczkowski. 2012. Making sense of the newspaper crisis: A critical assessment of existing research and an agenda for future work. New Media \& Society 14, 8: 1375-1394. [31] B. Smith. 2017. How tech and media can fight fake news. Colombia Journalism Review.

[32] Snap Inc. 2015. Introducing Discover. Retrieved from https://www.snap.com/enUS/news/post/introducing-discover/

[33] Snap Inc. 2017. Snapchat Privacy Policy. Retrieved April 12, 2017 from

https://www.snap.com/en-GB/privacy/privacy-policy/ [34] Anselm Strauss and Juliet Corbin. 1994. Grounded Theory Methodology: An Overview Handbook of Qualitative Research. In Handbook of qualitative research. 273-285.

[35] Karine Barzilai-nahon. 2005. Network Gatekeeping Theory. Pre-print Version.

[36] Bin Xu, Pamara Chang, Christopher L Welker, Natalya N Bazarova, and Dan Cosley. 2016.

Automatic Archiving versus Default Deletion: What Snapchat Tells Us About Ephemerality in Design. CSCW'16, 1660-1673.

[37] V. Zakem. 2017. How Russia's Disinformation Campaign Could Extend Its Tentacles. National Public Radio. 